\title{
Personal and Perceived Depression Stigma in Individuals Affected by Depression and Relatives: Results of a Survey among Attendees of a German Depression Congress
}

\author{
Heinz $I^{1 *}$, Mergl $R^{2 * \#}$, Allgaier A-K ${ }^{2}$, Hegerl $U^{3}$ and \\ Rummel-Kluge $\mathrm{C}^{1}$ \\ ${ }^{1}$ Department of Psychiatry and Psychotherapy, Medical \\ Faculty, University of Leipzig, Germany \\ ${ }^{2}$ Institute of Psychology, Universität der Bundeswehr \\ München, Germany \\ ${ }^{3}$ Department of Psychiatry, Psychosomatic Medicine and \\ Psychotherapy, University Hospital Frankfurt, Germany \\ \#This Author Equally Contributed to this Manuscript \\ *Corresponding author: Mergl R, Institute of \\ Psychology, Universität der Bundeswehr München, \\ Werner-Heisenberg-Weg 39, D-85577 Neubiberg, \\ Germany
}

Received: July 28, 2021; Accepted: August 11, 2021; Published: August 18, 2021

\begin{abstract}
Background: Depression stigma is a clinically relevant factor negatively affecting the help-seeking process and depression care. Relatives of individuals suffering from depression play an important role in service utilization and in depression treatment, but little is known about their depression stigma compared to the stigma of individuals affected.
\end{abstract}

Aims: We investigated whether individuals with depression, relatives and individuals being both - affected and relative - differ in depression stigma.

Methods: Paper-pencil questionnaire data of 216 study participants from a German depression congress in 2017 were analyzed using Kruskal-Wallis tests to investigate subgroup differences and Mann-Whitney-U tests for post-hoc comparisons. Ordinal logistic generalized regression models with the dependent variables being the stigma sum scores and the independent variables "group", "gender" and "age" were computed.

Results: Participants being a relative of an individual with depression, being affected by depression or being both - relative and affected - reported comparable personal and perceived depression stigma. There was a statistical trend for group differences in personal stigma in the total sample, due to significantly lower personal stigma in male participants being affected by depression compared to male participants having a family member affected.

Conclusions: Relatives of individuals with depression appear to have similar stigmatizing attitudes as affected individuals themselves. Potential differences in personal stigma in male relatives compared to male patients require further research, since they have implications for anti-stigma activities as well as for depression care.

Keywords: Depression; Stigma; Attitudes; Relatives; Family members

\section{Abbreviations}

B: participants with depression and being relative; D: participants with depression; DSS: Depression Stigma Scale; DSS-perceived: Depression Stigma Scale-sum score for perceived depression stigma; DSS-personal: Depression Stigma Scale-sum score for personal depression stigma; DSS-total: Depression Stigma Scale-sum score for personal and perceived depression stigma; M: (arithmetical) means; $\mathrm{N}$ : sample size; $\mathrm{n}$ : subgroup size; R: participants being relatives of individuals with depression; SD: Standard Deviation; WHO: World Health Organization

\section{Introduction}

Mental health stigma has an impact on psychological, physiological and economic outcomes of individuals affected [14]. Stigma is one of the major barriers to recognize a mental health problem, to seek professional help and to receive adequate treatment [5-13]. The most established definition of stigma is Goffman's [14] describing stigma as an "attribute that is deeply discrediting" (p. 3) and linked to stereotypes, that devalues an individual. Common attributes for individuals with depression are being "unpredictable", "dangerous" $[15,16]$, "weak" and not motivated to overcome the depression-attributes that reflect pretty much the opposite of what our society considers as the norm [17].

In addition to Goffman, other concepts of stigma have been developed and different types of stigma are described in the literature. Corrigan for example distinguishes between public stigma referring to the broad public's attitudes towards a stigmatized person and self- or internalized stigma, which describes the internalized public stigma by an individual of the stigmatized group [18]. Griffiths and colleagues differentiate between personal stigma, an individual's personal believes about depression (e.g. 'Depression is not a real medical illness') and perceived stigma (e.g. 'Most people believe that depression is not a real medical illness') [19]. Perceived stigma is related to public stigma but reflects how much an individual perceives public attitudes towards depression.

A validated and widely used instrument to measure personal and perceived depression stigma is the Depression Stigma Scale (DSS), developed by Griffiths [19]. Numerous studies have shown that when
Ann Depress Anxiety - Volume 8 Issue 1 - 2021

ISSN : 2381-8883 | www.austinpublishing group.com

Mergl et al. ( ) All rights are reserved
Citation: Heinz I, Mergl R, Allgaier A-K, Hegerl U and Rummel-Kluge C. Personal and Perceived Depression Stigma in Individuals Affected by Depression and Relatives: Results of a Survey among Attendees of a German Depression Congress. Ann Depress Anxiety. 2021; 8(1): 1106. 
measured with the DSS, scores for perceived stigma are higher than those for personal stigma [11,20-22]. Furthermore, personal and perceived stigma scores vary between different groups depending on e.g. gender, age, personal history of depression as well as the level of contact to individuals with depression (e.g. having a family member affected) [1,6,11,20,23-25]. The stigma of relatives of individuals suffering from depression is of great interest, since relatives can impact on how patients deal with their illness, for instance they can refer them to seek professional help [26] or be opposed to it [27]. Moreover, it is often the family members who communicate with mental health professionals. As key persons especially when giving care to affected individuals, they understand the patient the most and thus can support mental health professionals in treating depression [28], e.g. in supporting pharmacological treatment adherence [29]. A family member's stigma may therefore impede a patient's recovery.

Previous studies adressing stigma in relatives or family members of individuals with depression mainly compared their personal and perceived stigma to that hold by members of the general population. For perceived stigma, higher scores have been found in both relatives of individuals suffering from depression as well as in affected individuals themselves as compared to the general public $[1,20,21,23,25]$. Experiencing more stigmatizing events and a greater sensitivity to those events are among the factors discussed for this association in depressed individuals. Likewise, the personal contact to an affected person e.g. within the family appears to be associated with a greater exposure to stigmatizing events, too [21]. Regarding personal stigma, findings are somewhat inconsistent. Some researchers revealed lower personal stigma scores for individuals with depression as well as for relatives of individuals with depression compared to the general public $[6,11,20,23,24]$ arguing that experiencing the disorder first hand or by a family member increases the understanding of the condition and the respective tolerance [20] or may be associated with an increased knowledge about depression or both [11]. Other studies did not reveal differences in personal stigma (assessed with the DSS) between patients, relatives, and the broad public $[15,21]$. However, only a few studies compared depression stigma between individuals with depression and relatives of individuals with depression revealing comparable personal and perceived stigma scores for both groups [21]. This comparison is of at least as much importance as the comparison of these groups with general population samples, because of the closer relationship of a relative to a depressed individual and the relative's involvement in depression care. Based on findings from a former study with a similar sample [21], the aim of this study was to investigate whether three groups of attendees of a German depression congress (participants with depression, participants being a relative of an individual with depression and participants being both affected and relative) significantly differ in personal and perceived depression stigma. Further we investigated if potential effects are moderated by relevant covariates such as age and gender, since covariates were not investigated so far, and that information might be important to tailor group-specific interventions to address stigma. We hypothesized according to [21] that participants with depression, participants being a relative of an individual with depression and participants being both affected and relative do not differ in personal and perceived stigma. Further, it was examined in an explorative way whether potential differences in personal or perceived stigma between these three subgroups were moderated by age and gender.

\section{Materials and Methods}

\section{Sample}

The study is based on data from a German sample, i.e. attendees of the German Depression Congress in Leipzig, Germany in the year 2017. Attendees came from all over Germany and were mainly affected by depression themselves and/or relatives of individuals with depression. The remaining participants were primarily interested in the topic neither being affected nor having an affected family member. All attendees had been asked to complete a paper-pencil questionnaire on site for evaluation purposes. The survey received a positive vote of the ethical committee of the Medical Faculty at the University of Leipzig, Germany (Reference number: 205/13-ek) and was conducted according to the principles of the Declaration of Helsinki. All participants gave written informed consent prior to the survey.

\section{Instrument}

Participants filled in the standardized Depression Stigma Scale (DSS) $[19,22,23]$ containing two sub-scales with 9 items each to assess personal depression stigma (DSS-personal) and perceived depression stigma (DSS-perceived) on a five-point Likert scale ranging from ,strongly disagree' (0) to 'strongly agree' (4). Higher sum scores on each scale (range 0-36) and in total indicate more stigmatizing attitudes towards depression. The questionnaire was presented in a German version. Forward translation as well as back-translation of the original English version by Griffiths [19] were performed following the guidelines published by the World Health Organization (WHO) [30]. The DSS demonstrated sufficient to good internal consistency for the two subscales and high test-retest reliability for the DSS as a whole $[8,19,23]$. Participants further provided sociodemographic information on age, gender, educational level, and status (individual with depression, relative of an individual with depression, individual being both affected and relative, individual being neither affected nor relative). We intentionally kept the survey short and did not, for example, use questionnaires assessing symptoms of depression so as not to give the impression that research was the primary concern at the event.

\section{Statistical analysis}

In order to test subgroup differences (defined by "status") in DSSpersonal and DSS-perceived a Kruskal-Wallis test was performed. By using the Mann-Whitney-U-Test, differences between two groups of participants (e.g., participants with depression versus relatives of an individual with depression) regarding DSS-personal and DSSperceived stigma were tested for statistical significance. These tests were not only conducted for the total sample but also for subgroups stratified by gender and age group. Moreover, we selected ordinal logistic generalized regression models with the dependent variables being DSS-personal and DSS-perceived and the independent variables "status" (three categories: 1: participant with depression, 2: participant being relative of an individual with depression, 3: participant being both affected and relative; reference category: 3 ), "gender" (two categories: 1: female, 2: male; reference category: 2) and "age group" (based on the terciles of the age distribution in the final sample leading to three categories: 1: not exceeding 39 years, 
2: 40 to 54 years, and 3: at least 55 years; reference category: 3) in order to be able to separate effects of the factor "status" from effects of other variables with a potential influence on DSS-personal and DSSperceived. This model was chosen rather than a general linear model since DSS items were rank scaled. Further, two-fold interactions of the factor "status" and the two factors "gender" and "age group" had been analyzed to identify potential moderators of status effects on depression stigma. The level of significance was defined as $\alpha=0.05$. Only in the case of post-hoc group comparisons an alpha-adjustment according to the Bonferroni correction was applied. All statistical tests were two-sided. Effect sizes were interpreted according to Cohen [31].

\section{Results}

\section{Sample characteristics}

Out of $N=271$ participants who gave their written informed consent, 249 (91.9\%) filled in the questionnaires. Due to missing data regarding age and in DSS items or inconsistent data regarding sex, 23 (8.49\%) participants were excluded. From the remaining 226 participants $(83.39 \%)$, only ten $(4.4 \%)$ were neither affected by depression nor relative of an affected individual. With regard to the low statistical power, this subgroup was excluded. Thus, the final sample consisted of 216 participants; characteristics are summarized in Table 1.

The final sample $(N=216)$ and the group of excluded participants ( $N=55$; due to missing/inconsistent values, or status "being neither depressed nor relatives of an individual with depression") were

Table 1: Description of the sample.

\begin{tabular}{|l|c|}
\hline \multicolumn{1}{|c|}{ Variables } & Final sample (N=216) \\
\hline Gender & $166(76.9 \%)$ \\
\hline Females & $50(23.1 \%)$ \\
\hline Males & \\
\hline Age group & $63(29.2 \%)$ \\
\hline Not exceeding 39 years & $77(35.6 \%)$ \\
\hline 40 to 54 years & $76(35.2 \%)$ \\
\hline At least 55 years & $(n=215)$ \\
\hline Educational level ${ }^{\text {a }}$ & $22(10.2 \%)$ \\
\hline No graduation & $61(28.4 \%)$ \\
\hline Academic or vocational education & $128(59.5 \%)$ \\
\hline At least graduate degree (like Bachelor of Arts) & $4(1.9 \%)$ \\
\hline Other & $50(23.1 \%)$ \\
\hline Status & $38(17.6 \%)$ \\
\hline Participant with depression & --- \\
\hline (99 females (77.3\%), 29 males (22.7\%)) & $128(59.3 \%)$ \\
\hline $\begin{array}{l}\text { Participant being relative of an individual with } \\
\text { depression }\end{array}$ & \\
\hline (24 females (63.2\%), 14 males (36.8\%)) & \\
\hline Participant being both affected and relative & \\
\hline (43 females (86.0\%), 7 males (14.0\%)) & \\
\hline & \\
\hline
\end{tabular}

Notes: ${ }^{a}$ For one individual information about the educational level was not available. Excluded individuals: Subjects with inconsistent values in gender or missing values in items of the Depression Stigma Scale (DSS), age or status "being neither depressed nor relatives of an individual with depression". comparable regarding the gender distribution and educational level. Excluded individuals were older than persons from the final sample only by trend $(Z=-1.84 ; p=0.07)$. Significant differences between excluded and included individuals in status distribution were due to the exclusion of persons being neither affected by depression nor relatives of an individual affected. If this subgroup is excluded from the analysis the status differences are no longer significant $\left(\chi^{2}=0.58\right.$; $d f=2 ; p=0.75)$.

\section{Personal and perceived stigma}

Table 2 summarizes the mean values for the DSS sum scores both in the total sample and several subgroups stratified by status, gender, and age.

Overall, sum scores for DSS-personal were significantly lower than corresponding scores for DSS-perceived (mean difference $=$ -16.22; standard deviation (SD) $=7.87 ; Z=-12.47 ; p<0.0001$; effect size $r=0.60$ ). There was a statistical trend for status differences regarding DSS-personal $(H=4.90 ; d f=2 ; p=0.09)$, with participants affected by depression demonstrating significantly lower sum scores than participants being relatives of an individual affected at the 5\% level $(Z=-2.233$; $p=0.03$; effect size $r=0.17)$. Other significant status differences regarding DSS sum scores did not occur. Neither gender differences nor age group differences for DSS sum scores were statistically significant. The ordinal logistic generalized regression models to analyze status effects on DSS-personal and DSS-perceived as well as the potential moderating effects of the factors "gender" and "age" revealed no significant results. Regarding DSS-personal, statusrelated differences between the corresponding DSS sum scores were only significant by trend $\left(\chi^{2}=5.96 ; d f=2 ; p=0.051\right)$ due to significant differences between participants with depression (D) and participants being relatives of individuals with depression $(\mathrm{R})(\mathrm{D}<\mathrm{R}$; see Table 2$)$ and neither moderated by the factor "age group" nor by the factor "gender". Regarding the DSS-perceived, there was no significant association with status, age group, and gender. The status differences of these scores were not moderated by age or gender.

Table 3 summarizes status differences regarding DSS sum scores for the total sample and several sub-samples stratified by gender and age.

According to results of the Kruskal-Wallis tests, there was a statistical trend for status differences in DSS-personal in the subgroup of males $(H=5.81 ; d f=2 ; p=0.055)$, due to significantly lower mean scores for DSS-personal in individuals with depression as compared to relatives of individuals with depression $(Z=-2.43$; $p=0.015)$. This finding was significant at the alpha-adjusted significance level $(\alpha=$ $0.017)$ and associated with a medium effect size $(r=0.37)$. There were neither significant status differences for DSS-perceived in the total sample nor in subgroups defined by gender and age.

\section{Discussion}

Individuals with depression, relatives of individuals with depression and individuals being both affected and relative did not differ in personal and perceived depression stigma (according to hypothesis 1). These results are in line with a previous study on personal and perceived depression stigma of a similar sample [21] and former studies showing lower personal stigma and higher perceived stigma are equally associated with having an own history of 
Table 2: Depression stigma sum scores for the total sample and subgroups.

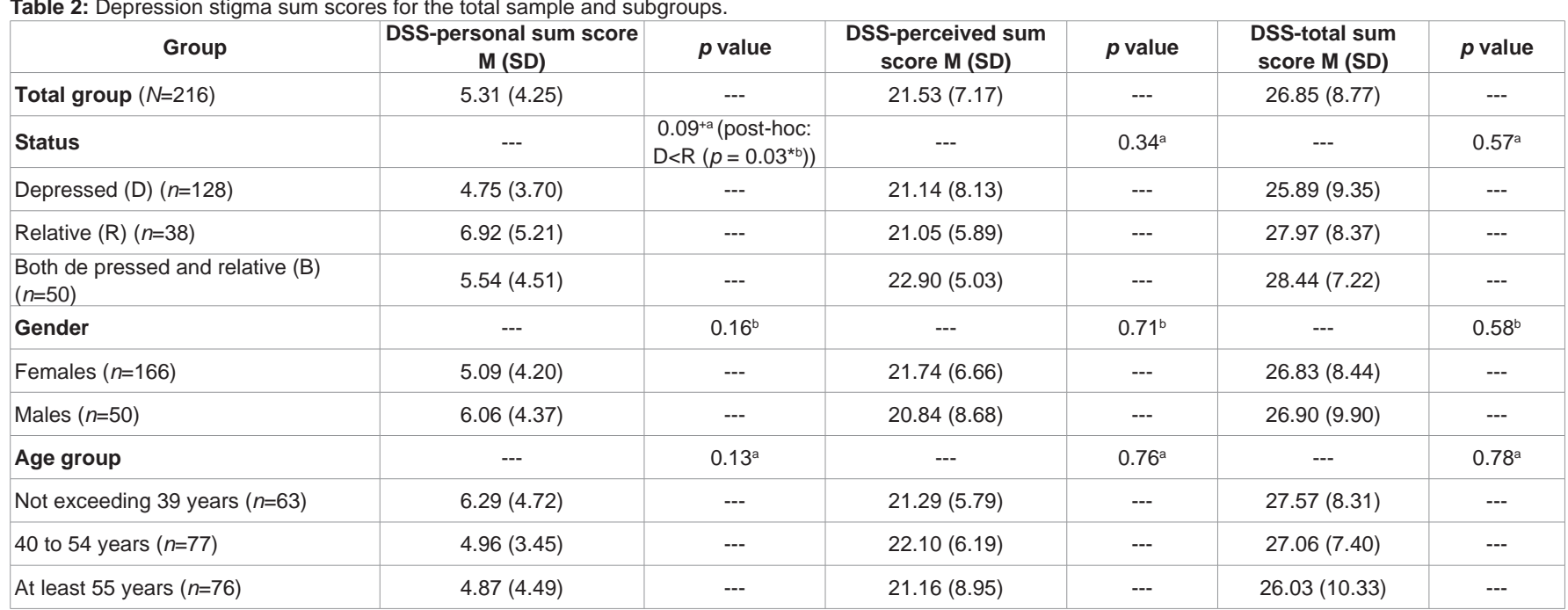

Notes: $M=$ means; $N=$ sample size; $n=$ subgroup size; $S D=$ standard deviation; DSS-personal = sum score personal depression stigma (maximum possible score: 36 ); DSS-perceived = sum score perceived depression stigma (maximum possible score: 36 ); DSS-total = sum score personal and perceived depression stigma (maximum possible score: 72 ); Depressed $(D)=$ participants with depression; Relative $(R)=$ participants being relative of an individual with depression; $(B)=$ participants with depression and being relative. ${ }^{+} p<0.10 ;{ }^{*} p<0.05$.

aaccording to the result of a Kruskal-Wallis test;

baccording to the result of a Mann-Whitney $U$ test.

Table 3: Differences in personal and perceived depression stigma in relation to status.

\begin{tabular}{|c|c|c|c|c|}
\hline \multirow{2}{*}{ Variables } & $\mathbf{D}$ & $\mathbf{R}$ & $\mathbf{B}$ & \multirow{3}{*}{$\mathbf{p}^{\mathbf{a}}$} \\
\cline { 2 - 4 } & $(n=128)$ & $(n=38)$ & $(n=50)$ & $(M, S D)$ \\
\cline { 2 - 3 } & $(M, S D)$ & $(M, S D)$ & Post-hoc comparisons \\
& &
\end{tabular}

Females $(n=166)$

\begin{tabular}{|c|c|c|c|c|c|}
\hline$n$ & 99 & 24 & 43 & -- & -- \\
\hline DSS-personal & $4.78(3.89)$ & $5.96(5.03)$ & $5.33(4.40)$ & 0.64 & -- \\
\hline DSS-perceived & $21.51(7.78)$ & $20.96(4.59)$ & $22.72(4.49)$ & 0.39 & -- \\
\hline
\end{tabular}

Males $(n=50)$

\begin{tabular}{|c|c|c|c|c|c|}
\hline$n$ & 29 & 14 & 7 & -- & - \\
\hline DSS personal & $4.66(3.03)$ & $8.57(5.27)$ & $6.86(5.27)$ & 0.06 & $\mathrm{D}<\mathrm{R} p=0.015^{*}$ \\
\hline DSS perceived & $19.90(9.29)$ & $21.21(7.83)$ & $24.00(8.00)$ & 0.48 & - \\
\hline
\end{tabular}

$\leq 39$ years $(n=63)$

\begin{tabular}{|c|c|c|c|c|c|}
\hline$n$ & 34 & 10 & 19 & --- & -- \\
\hline DSS-personal & $5.74(4.51)$ & $8.90(5.41)$ & $5.89(4.51)$ & 0.19 & -- \\
\hline DSS-perceived & $19.62(6.50)$ & $23.40(4.88)$ & 23.16 (3.83) & 0.11 & --- \\
\hline \multicolumn{6}{|l|}{$40-54$ years $(n=77)$} \\
\hline$n$ & 50 & 8 & 19 & --- & --- \\
\hline DSS-personal & $4.72(3.33)$ & $5.63(3.38)$ & $5.32(3.87)$ & 0.8 & --- \\
\hline DSS-perceived & $21.56(7.08)$ & $21.75(2.32)$ & $23.68(4.46)$ & 0.41 & --- \\
\hline \multicolumn{6}{|l|}{$\geq 55$ years $(n=76)$} \\
\hline$n$ & 44 & 20 & 12 & --- & --- \\
\hline DSS-personal & $4.02(3.30)$ & $6.45(5.63)$ & $5.33(5.68)$ & 0.31 & --- \\
\hline DSS-perceived & $21.84(10.16)$ & $19.60(6.99)$ & $21.25(7.20)$ & 0.26 & --- \\
\hline
\end{tabular}

Notes: $M=$ (arithmetical) mean; $N / n=$ (sub-)sample size; $S D=$ standard deviation; DSS-personal = sum score personal depression stigma; $D S S$-perceived = sum score perceived depression stigma; $\mathrm{D}=$ participants with depression; $\mathrm{R}=$ participants being relative of an individual with depression; $\mathrm{B}=$ both affected and relative. abased on a Kruskal-Wallis test;

bbased on Mann-Whitney $U$ tests (only statistically significant findings are reported here (with the significance level being alpha $=0.05$ );

*significant at the alpha-adjusted significance level $(\alpha=0.017(=0.05 / 3))$. 
depression, a parental history of depression or prior contact to people with depression $[6,20,23,25]$.

Further, depression stigma seems not to be higher or lower when being both affected by depression as well as a relative of an affected person. Our findings imply that individuals with depression as well as their relatives benefit equally from interventions to reduce stigma, without tailoring them to these respective subgroups. However, the additional explorative analysis of status-related group differences in stigma, stratified regarding age and gender, revealed a significantly lower personal depression stigma in males with depression as compared to male relatives (medium effect size). Several studies demonstrated gender differences in personal stigma for males showing higher scores than females (e.g. $[6,20,23])$ concluding less awareness, less mental health literacy or lower understanding for the disorder in males [32], possibly due to a lower prevalence of depressive disorders in men than in women [33]. In our sample, personal stigma was comparable between males and females, only the subgroups of male relatives of individuals with depression reported significantly higher personal stigma compared to male individuals with depression. A possible explanation for this difference could be that male depressed individuals have more understanding of the condition due to experiencing depression themselves. Male relatives - on the other hand - may have more difficulties to accept the condition of an affected family member because they lack this first-hand experience. Females may compensate this lack through a higher empathy, awareness of depression and/or a higher knowledge about the disorder [34]. This could explain why contact with a family member or close friend suffering from depression is associated with lower personal stigma in women, but not in men as also being demonstrated in [34]. However, whether personal stigma in males differs depending on the status, i.e. experiencing depression "firsthand" or "only" by a family member requires further research with a representative sample. If so, clinicians should be aware of male relatives and help them to overcome stigmatizing attitudes and thereby enhancing family support.

It should be noted that the overall low scores for personal stigma with a mean of $5.31(S D=4.25)$ for the total sample are striking. It is likely that only individuals with favourable attitudes and knowledge about depression were open to attend such a public event on a mental health topic that is accompanied by strong media coverage. That might also explain, why we did not find differences in personal stigma between females and males as previously reported. Individuals with higher personal stigma may be less likely to potentially disclose their own (or a family member's) depression by attending such an event or even before getting the depression diagnosis - don't appraise depression symptoms as part of a mental disorder [12]. Path models of help seeking showed that personal stigma, in particular, influences the perception and appraisal of depressive symptoms. This "selfidentification" as being affected from a mental health problem seems to be crucial and also impacts subsequently, how a person deals with the symptoms, e.g. whether to seek help [13]. This should be considered when planning activities to raise awareness on a mental health disorder and to address stigma. Public events may attract mainly people that already have a connection to the topic, i.e. having identified themselves as affected by depression or having already received a diagnosis $[12,13]$. It is conceivable that this also applies to relatives. Therefore, such events alone may not be sufficient to reach out to individuals with higher stigma, albeit they provide the chance for personal contact that is found to reduce stigmatising attitudes and increases help-seeking intentions $[3,4,35]$.

The strengths of the present study are a rather high sample size allowing moderator analyses and the application of the DSS being a standardized instrument. However, there are some methodological limitations restricting the validity and generalizability of the reported findings. First, the present study refers to attendees of a public congress and it can be assumed that their stigma scores are not representative for the stigma of individuals affected and relatives, who did not attend the event. Also, the small number of male participants is a methodological limitation. Second, we cannot exclude that social desirability had influenced the answers in the DSS. Third, it would have been interesting to address other relevant predictors of depression stigma like previous experiences with antistigma campaigns targeting depression $[21,36]$ as well as beliefs in a continuum "from mentally healthy to ill" [37]. Fourth, depression diagnosis of participants based on self-report and measures of the severity of current depressive symptoms had not been applied. Likewise, the status "suffering from depression" or "being a relative" or "being both" based on self-report. Future research should take unambiguous definitions of "relatives" into account to increase comparability. Some studies merely assess "contact to a person with depression" (e.g. [24]), others assess parental history of depression (e.g. [20]) or ask specifically for family members (e.g. [23]) or family members and close friends (e.g. [6,34]), while we assessed according to Dietrich and colleagues $[11,21]$ three categories labeled "patient", "relative", and "patient and relative".

\section{Conclusion}

In conclusion, individuals with depression, relatives of individuals with depression and individuals being both depressed and relative reported comparable personal stigma and perceived stigma. While status-related group differences in depression stigma were neither moderated by age nor by gender, subgroup analyses revealed a significant group difference in personal stigma between male participants with depression and male relatives, the latter showing significantly higher personal stigma. This finding is relevant in view of the fact that relatives play an important role regarding service utilization for depression by affected patients [26] and in depression treatment $[28,29]$. Research now needs to be extended using representative populations of relatives of individuals with depression, since findings on gender differences in this subpopulation have implications for anti-stigma-interventions and care providers.

\section{Acknowledgment}

Parts of this manuscript were prepared within the context of Robert Leuoth's Bachelor Thesis at the Institute of Psychology, Universität der Bundeswehr München. We wish to thank Prof. Schomerus for his constructive input to the manuscript.

\section{References}

1. Grant JB, Bruce CP, Batterham PJ. Predictors of personal, perceived and self-stigma towards anxiety and depression. Epidemiol Psychiatr Sci. 2016; 25: $247-254$

2. Lasalvia A, Zoppei S, van Bortel T, et al. Global pattern of experienced and 
anticipated discrimination reported by people with major depressive disorder: a cross-sectional survey. Lancet. 2013; 381: 55-62.

3. Rüsch N, Angermeyer MC, Corrigan PW. Mental illness stigma: concepts consequences, and initiatives to reduce stigma. Eur Psychiatry. 2005; 20 529-539.

4. Schomerus $\mathrm{G}$, Matschinger $\mathrm{H}$, Angermeyer MC. The stigma of psychiatric treatment and help-seeking intentions for depression. Eur Arch Psychiatry Clin Neurosci. 2009; 259: 298-306.

5. Clement S, Schauman O, Graham T, et al. What is the impact of mental health-related stigma on help-seeking? A systematic review of quantitative and qualitative studies. Psychol Med. 2015; 45: 11-27.

6. Coppens E, van Audenhove C, Scheerder G, et al. Public attitudes toward depression and help-seeking in four European countries baseline survey prio to the OSPI-Europe intervention. J Affect Disord. 2013; 150: 320-329.

7. Barney LJ, Griffiths KM, Jorm AF, Christensen H. Stigma about depression and its impact on help-seeking intentions. Aust NZ J Psychiatry. 2006; 40: 51-54.

8. Griffiths KM, Crisp DA, Jorm AF, Christensen $\mathrm{H}$. Does stigma predict a belief in dealing with depression alone? J Affect Disord. 2011; 132: 413-417.

9. Sirey JA, Bruce ML, Alexopoulos GS, Perlick DA, Friedman SJ, Meyers BS Stigma as a barrier to recovery: Perceived stigma and patient-rated severity of illness as predictors of antidepressant drug adherence. Psychiatr Serv. 2001; 52: 1615-1620.

10. Sirey JA, Bruce ML, Alexopoulos GS, et al. Perceived stigma as a predicto of treatment discontinuation in young and older outpatients with depression. Am J Psychiatry. 2001; 158: 479-481.

11. Dietrich S, Mergl R, Rummel-Kluge C. Personal and perceived stigmatization of depression: a comparison of data from the general population, participants of a depression congress and job placement officers in Germany. Psychiatry Res. 2014; 220: 598-603.

12. Horsfield P, Stolzenburg S, Hahm S, et al. Self-labeling as having a mental or physical illness: the effects of stigma and implications for help-seeking. Soc Psychiatry Psychiatric Epidemiol. 2020; 55: 907-916.

13. Schomerus G, Stolzenburg S, Freitag S, et al. Stigma as a barrier to recognizing personal mental illness and seeking help: a prospective study among untreated persons with mental illness. Eur Arch Psychiatry Clin Neurosci. 2019; 269: 469-479.

14. Goffman E. Stigma: Notes on the management of spoiled identity. New Jersey: Prentice-Hall. 1963

15. Wang J, Lai D. The relationship between mental health literacy, personal contacts and personal stigma against depression. J Affect Disord. 2008; 110 191-196.

16. Economou M, Bergiannaki JD, Peppou LE, et al. Attitudes towards depression, psychiatric medication and help-seeking intentions amid financial crisis: Findings from Athens area. Int J Soc Psychiatry. 2016; 62: 243-251.

17. Baer N, Sikorski C, Luppa M, Riedel-Heller SG, Schomerus G. Das Stigma Depression-eine Interaktion zwischen öffentlichem Diskurs und Erfahrungsberichten Betroffener. Psychiatr Prax. 2016; 43: e1-e8.

18. Corrigan P. How stigma interferes with mental health care. Am Psychol. 2004; 59: 614-625.

19. Griffiths KM, Christensen H, Jorm AF, Evans K, Groves C. Effect of web based depression literacy and cognitive-behavioural therapy interventions on stigmatising attitudes to depression: randomised controlled trial. $\mathrm{Br}$ Psychiatry. 2004; 185: 342-349.
20. Calear AL, Griffiths KM, Christensen H. Personal and perceived depression stigma in Australian adolescents: magnitude and predictors. J Affect Disord. 2011; 129: 104-108.

21. Dietrich S, Mergl R, Rummel-Kluge C. Von den ersten Symptomen bis zur Behandlung einer Depression. Wann und bei wem suchen Menschen mit Depression Hilfe? Welche Rolle spielt Stigmatisierung? Psychiatr Prax. 2017; 44: 461-468.

22. Griffiths KM, Nakane Y, Christensen H, Yoshioka K, Jorm AF, Nakane H. Stigma in response to mental disorders: a comparison of Australia and Japan. BMC Psychiatry. 2006; 6: 21.

23. Griffiths KM, Christensen $\mathrm{H}$, Jorm AF. Predictors of depression stigma. BMC Psychiatry. 2008; 8: 25.

24. Boerema AM, van Zoonen $\mathrm{K}$, Cuijpers $\mathrm{P}$, et al. Psychometric properties of the Dutch Depression Stigma Scale (DSS) and associations with personal and perceived stigma in a depressed and community sample. PLoS One. 2016; 11: e0160740.

25. Pyne JM, Kuc EJ, Schroeder PJ, Fortney JC, Edlund M, Sullivan G. Relationship between perceived stigma and depression severity. J Nerv Ment Dis. 2004; 192: 278-283.

26. Dew MA, Bromet EJ, Schulberg HC, Parkinson DK, Curtis EC. Factors affecting service utilization for depression in a white collar population. Soc Psychiatry Psychiatr Epidemiol. 1991; 26: 230-237.

27. Shi W, Shen Z, Wang S, Hall BJ. Barriers to professional mental health helpseeking among Chinese adults: A systematic review. Front Psychiatry. 2020; 11: 442

28. Shi $\mathrm{Y}$, Shao $\mathrm{Y}$, Li H, et al. Correlates of affiliate stigma among family caregivers of people with mental illness: A systematic review and metaanalysis. J Psychiatr Ment Health Nurs. 2019; 26: 49-61.

29. Bollini P, Pampallona S, Kupelnick B, Tibaldi G, Munizza C. Improving compliance in depression: a systematic review of narrative reviews. J Clin Pharm Ther. 2006; 31: 253-260.

30. World Health Organization. Process of translation and adaptation of instruments. Geneva: World Health Organization. 2014

31. Cohen J. Statistical power analysis for the behavioral sciences. $2^{\text {nd }}$ edtion. Hillsdale, N.J.: L. Erlbaum Associates. 1988.

32. Cook TM, Wang J. Descriptive epidemiology of stigma against depression in a general population sample in Alberta. BMC Psychiatry. 2010; 10: 29.

33. Wright A, Jorm AF. Labels used by young people to describe menta disorders: Factors associated with their development. Aust NZ J Psychiatry. 2009; 43: 946-955

34. Wang J, Fick G, Adair C, Lai D. Gender specific correlates of stigma toward depression in a Canadian general population sample. J Affect Disord. 2007; 103: $91-97$

35. Wechsler D, Schomerus G, Mahlke C, Bock T. Effektivität eine kontaktbasierten Kurzintervention zum Abbau stigmatisierender Einstellungen unter MedizinstudentInnen: Ergebnisse einer randomisierten, kontrollierten Studie. Neuropsychiatr. 2020; 34: 66-73.

36. Quinn N, Knifton L, Goldie I, et al. Nature and impact of European anti-stigma depression programmes. Health Promot Int. 2014; 29: 403-413.

37. Schomerus G, Matschinger H, Angermeyer MC. Continuum beliefs and stigmatizing attitudes towards persons with schizophrenia, depression and alcohol dependence. Psychiatry Res. 2013; 209: 665-669. 